\title{
Two-state CDMA Reception for Satellite UMTS
}

\author{
Lorenzo Mucchi ${ }^{\ddagger}$, Luca Simone Ronga ${ }^{\dagger}$, Enrico Del Re ${ }^{\ddagger}$ \\ $\ddagger$ University of Florence \\ Department of Electronics and Telecommunications \\ Via di Santa Marta 3, 1-50139 - Florence, Italy. \\ E-mail:\{mucchi, delre\}@lenst.det.unifi.it \\ $\ddagger$ CNIT Florence Unit - University of Florence \\ Via di Santa Marta 3, 50139 - Florence, Italy. \\ E-mail: ronga@lenst.det.unifi.it
}

\begin{abstract}
In the third generation communication system, due to the average increase of the radio link bandwidth requirements and the power constraints at the mobile S-UMTS terminal, the system capacity will meet its physical limitations even in a moderated deployment scenario. In the 3GPP standard, due to the complexity constraints at the user equipment, the DTX implementation on the uplink channel is heavily limited. The transmission scheme presented in this paper is an extension of the traditional informative symbol set with a zero energy symbol. The silence symbols are integrated with the informative ones and delivered to the radio link layer for transmission. The paper presents a theoretical formulation of the optimum two-state receiver. Comparisons with the traditional single-states receiver are obtained with a theoretical approach and through computer simulations. In the latter case a real Globalstar-like SUMTS environment show the better performances of the proposed scheme for fast varying sources.

Keywords-CDMA System Performance, S-UMTS, Reciver and Transmitter Design, Signal Processing Applications in Telecommunications, Multiple Access Techniques.
\end{abstract}

\section{INTRODUCTION}

Bandwidth represents the last challenge in wireless personal communications. In the third generation communication system, due to the average increase of the radio link bandwidth requirements and the power constraints at the mobile S-UMTS terminal, the system capacity will meet its physical limitations even in a moderated deployment scenario.

The high data rate services included in the UMTS standard makes it difficult the coverage of large geographical areas with only terrestrial systems. The satellite segment is considered a necessary component to obtain the "anytime-anywhere"" UMTS service provision.

Every technology able to increase the spectral efficiency of the radio link maintaining the compatibility with the approved standards will play a fundamental role for the economical aspects of the UMTS diffusion.

Power consumption at the handheld terminal is another key issue. Mobile phones are requested to operate complex computational tasks at the expense of a reduced duration of the batteries. The techniques able to save energy by optimizing the transmission scheme play a fundamental role in the design phase.

The idea of a discontinued transmission has been exploited starting from the second generation of personal communication systems with the DTX feature in the GSM standard [1], [2], [3].

Voice activity detectors are designed to exploit the natural pauses in the speech flow to reduce the transmitted power and, consequently, battery life. Transmission silences for voice are

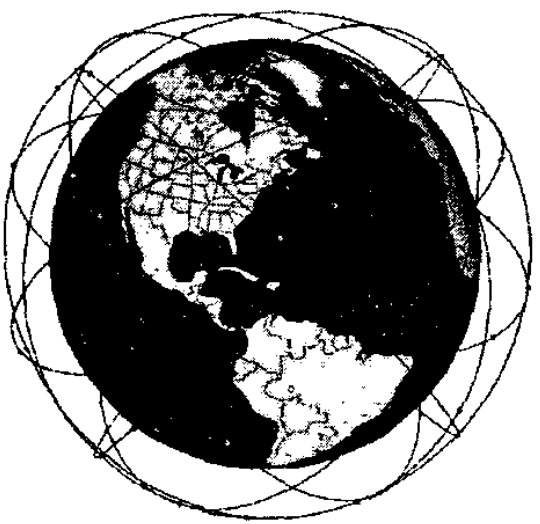

Fig. 1. Globalstar-like S-UMTS System

modeled as two states Markov chain where the average silences last for seconds [4]. There are however some information sources, like fast variable rate video coders or fast impulsive data sources which are characterized by very short silences, i.e. slotwise or even bitwise.

In the 3GPP standard, due to the complexity constraints. at the user equipment, the DTX implementation on the uplink channel is heavily limited. The silence state from the source is mapped on a true suppression of the transmitted signal only if the silence period is longer than a frame, i.e. $10 \mathrm{~ms}$. Thus the power saving only occurs for slow on-off transitions while fast VBR sources result in a continuous transmission at full rate.

Those considerations lead to the development of the transmission scheme presented in this paper. The basic idea is the extension of the traditional informative symbol set with a zero energy symbol. The silence symbols are integrated with the informative ones and delivered to the radio link layer for transmission [4]. The end-to-end signaling between the applications can be avoided and the radio layer does not need to receive any explicit transmit on/off commands from higher layers. The proposed reception scheme has also the property of being able to receive common single state transmissions. In this case, the silence symbols thresholds collapse to 0 and the receiver degenerates in a traditional single state receiver.

The advantages of the proposed solution can be summed up 
in the following list:

- the reduction of the average transmit power from a CDMA terminal, obtained by employing silence symbols, reduces the interference on other users,

- the radio layer need not to be integrated with the silence state management function of the application layer,

- silence symbols allow very short traffic bursts and a great variety of fractional bit-rates without increasing the MAI level.

A theoretical analysis of the performance of the proposed two-states CDMA receiver has been previously published in [5] and [6]. In this paper the proposed communication scheme has been moved into a real 3GPP S-UMTS environment and the performance of the two-states receiver has been taken via computer simulations.

The paper has been organized as follow: in section II the proposed two-states communication strategy is described and the optimum detector is derived. Section III reports the generalized probability of error for a two-states CDMA communication system. The simulated 3GPP S-UMTS environment is also described in the section. Numerical results and conclusions are shown in Section IV and Section V, respectively.

\section{CDMA Two-StATES RECEPTION.}

With the proposed scheme, the general base-band transmission signal of the $k$ th user is:

$$
\begin{gathered}
s_{k}(t)=\sum_{n=-\infty}^{n=\infty} s_{k}(t)^{(n)} \\
s_{k}(t)^{(n)}=A_{k} m_{k}^{\langle n\rangle} b_{k}^{\langle n\rangle} g_{k}^{\langle n\rangle}\left(t-n T_{s}\right)
\end{gathered}
$$

where

$$
g_{k}^{(n)}(t)==\sum_{i=1}^{G} c_{k}^{(n)}(i) p\left(t-i T_{c}\right)
$$

and

$T_{s}$ is the symbol time,

$T_{c}$ is the chip time,

$G=T_{s} / T_{\mathrm{c}}$ is the processing gain,

$A_{k}=\sqrt{E_{k}}$ the transmitted amplitude for user k,

$p(\dot{t})$ is the complex valued chip waveform due to pulse shaping filter,

$c_{k}^{(n)}$ is the $k$ th normalized spreading code of user $k$ referred to $n$th symbol interval,

$m_{k}^{(n)}$ is the mask symbol which assumes one of the two possible values $\{0,1\}$. It determines the state of the transmitter in the n-th time interval: Talk or Silent.

$b_{k}^{(n)}$ is the informative symbol transmitted during the $\mathrm{n}$-th interval, chosen among the symbol alphabet of the chosen modulation (e.g. for a BPSK signaling $b_{k}^{(n)} \in\{-1,1\}$ ). It has no significance when the transmitter is in the Silent state.
The received signal $r(t)$ expresses the observable part of the transmission chain. The received signal can be seen as:

$$
r(t)=\sum_{k=1}^{K} s_{k}(t)+n(t)
$$

where $n(t)$ is the white gaussian noise with zero mean and variance $\sigma^{2}$.

The unknown mask and symbol transmitted by the user over the transmission channel can be grouped in the two-state information symbol $q^{(n)}$ defined as:

$$
q^{(n)}=m^{(n)} b^{(n)}
$$

where we have dropped here the $k$ index for simplicity. The optimum detector [7], for a given set of transmitted twostate symbols will choose the symbol $\hat{q}^{(n)}$ corresponding to the largest posterior probability based on the observation of $r(t)$ (MAP criterion). Formally:

$$
\hat{q}^{(n)}=\arg \max _{q} P\left(q \mid r(t)^{(n)}\right)
$$

We can assume that the two-states are alternating independently from the informative stream, constituted by $M$ equally probable symbols. This leads to:

$$
\begin{gathered}
P\left(q_{\text {talk }}\right)=\frac{P(\text { talk })}{M} \\
P\left(q_{\text {silence }}\right)=1-P(\text { talk })
\end{gathered}
$$

where $P(t a l k)$ is the absolute probability of a talk symbol. The two-state symbol $q$ is thus possibly one of the equally probable $M$ informative symbols or the single "silence" one. The transmission model described above needs a more complex performance characterization with respect to the traditional one. The receiver is characterized by a general probability of error which is specialized in:

- probability of false detection of a silence state, $P_{\text {e,sil }}$

- probability of symbol error conditioned to a talk state, $P_{e, s y m b}$.

In the special case of a BPSK+ (the "plus" symbol indicates the presence of a "silent" state) operating on a AWGN channel, the optimum receiver is defined by the following thresholds:

$$
\begin{aligned}
& \theta_{0,2} \doteq \frac{\sigma^{2}}{\sqrt{E}} \ln \frac{P\left(q_{2}\right)}{P\left(q_{0}\right)}+\frac{\sqrt{E}}{2} \\
& \theta_{1,2} \doteq \frac{\sigma^{2}}{\sqrt{E}} \ln \frac{P\left(q_{1}\right)}{P\left(q_{2}\right)}-\frac{\sqrt{E}}{2}
\end{aligned}
$$

Where the symbols are labeled as in table I, and $E$ is the talk symbol energy.

The decision regions for the described receiver, with $h$ being the observable metric, are described by:

$$
\begin{cases}h<\theta_{1,2} & \text { the symbol } q_{1} \text { is selected } \\ \theta_{1,2} \leq h<\theta_{0,2} & \text { the symbol } q_{2} \text { is selected } \\ \theta_{0,2} \leq h & \text { the symbol } q_{0} \text { is selected }\end{cases}
$$


TABLE I

BPSK+ SIGNALING

\begin{tabular}{|c|c|c|}
\hline Symbol & Transmitter state & Informative symbol \\
\hline$q_{0}$ & Talk & 0 \\
$q_{1}$ & Talk & 1 \\
$q_{2}$ & Silent & n.a. \\
\hline
\end{tabular}

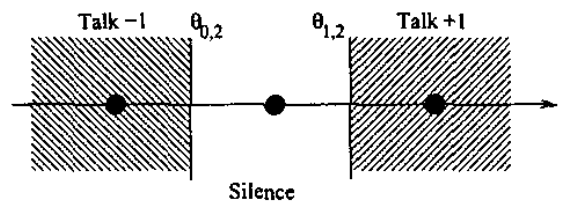

Fig. 2. BPSK+ Decision Regions

The decision regions are represented in Fig. 2.

\section{ERror and False Alarm Probability}

After each received symbol, the proposed two states reception scheme belongs to one of the possible states derived from the combination of both the transmitter and the receiver ones. This concept is explained in Table II. The columns represents the possible receiver decisions while the rows enumerate the transmitter symbols. The elements inside are called "conditions" of the whole reception system (i.e. transmitter + receiver).

The False Alarm condition is met when the Talk/Silence status is misinterpreted. An Error occurs when the transmitter is in the Talk state but the symbol is not correctly detected. The Correct condition is self explanatory. In order to provide the two states receiver with a performance index suitable for a comparison with the traditional reception, we consider the generalized probability of error or $P_{e g}$ defined as:

$$
P_{e g}=\operatorname{Pr} \text { (Error) } \cup \operatorname{Pr} \text { (False Alarm) }
$$

The cited index takes into account all the potential errors the receiver may commit when the transmitter is in the talk state. It should be noted that the two state system provides more information than the traditional "always on" reception, the additional information is transmitted at the expense of a reduced noise margin for the decision regions. This fact makes the comparison with the traditional system a difficult task.

In this paper th comparison has been carried out via computer simulations and the Bit Error Rate (BER) of the proposed

\begin{tabular}{c|ccc} 
& $\mathrm{Rx} q_{0}$ & $\mathrm{Rx} q_{1}$ & $\mathrm{Rx} q_{2}$ (silence) \\
\hline Tx $q_{0}$ & Correct & Error & False Alarm \\
$\operatorname{Tx} q_{1}$ & Error & Correct & False Alarm \\
$\operatorname{Tx} q_{2}$ & False Alarm & False Alarm & Correct: \\
& \multicolumn{4}{c}{ TABLE II } &
\end{tabular}

THE SET OF SYSTEM STATES two-states receiver has been evaluated with the same throughput of the traditional one-state receiver. A S-UMTS communication environment has been built following the 3GPP standard specifications [8].

To evaluate the probability of ternary symbol error, let us assume $\mathrm{K}$ users, $\mathrm{R}$ being the cross-correlation matrix and the threshold

$$
\begin{gathered}
\Phi=\theta_{0,2} \sqrt{E_{k}}=\sigma^{2} \ln \left(\frac{P\left(q_{2}\right)}{P\left(q_{0}\right)}\right)+\frac{E_{k}}{2} \\
\text { As shown in [5] and [6] the } P_{e g, \text { conv }}^{(2 s s)} \text { is: } \\
P_{e g, \text { conv }}^{(2 s)} \leq \sum_{z=0}^{K t}\left(\begin{array}{c}
K- \\
z
\end{array}\right) P_{t a l k}^{K-1-z}\left(1-P_{t a l k}\right)^{z} \\
Q\left(\frac{E_{k}-(K-1-z) \rho-\Phi}{\sqrt{E_{k} \sigma}}\right)
\end{gathered}
$$

where $\rho=\max _{j}\left\{\left|\mathbf{R}_{k j}\right|\right\}$ is the maximum element in the crosscorrelation matrix.

Analogously, the probability of error of a traditional onestate conventional detector can be written as [9]:

$$
P_{e, c o n v}^{(1 s)} \leq Q\left(\frac{E_{k}-(K-1) \rho}{\sigma \sqrt{E_{k}}}\right)
$$

The numerical comparison between eq. (12) and eq. (13) is reported in the Section IV.

A Globalstar-like satellite UMTS environment has been simulated. The Globalstar constellation guarantees two satellites in view at European latitudes, so that we can assume fairly that one of those satellites is always in the LOS state. If both of them are in the LOS state, we suppose to receive from the satellite that yields the higher SNR at the receiver (selection diversity).

Both DPDCH (data) and DPCCH (control) have been simulated although only data channel is considered in the BER calculation.

The two-states optimal threshold has been optimized numerically for the simulated environment. The optimal thresholds in (10) are related to the single user in AWGN channel. For the real S-UMTS scenario a numerical optimization has been executed to find the best decision region for each analyzed operating condition.

\section{NUMERICAL RESULTS}

First the dependence of the probability of generalized error from the operating point of the proposed CDMA communication scheme has been analyzed; the same operating condition have been then applied to the conventional single-state receiver and the resulting performances compared to those obtained by the proposed two-states communication scheme.

The comparisons reported in this document, however, do not take into account the additional information available at the 


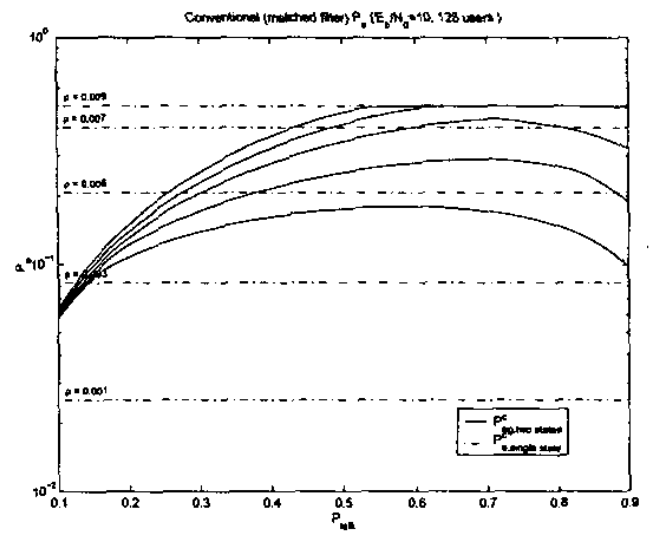

Fig. 3. $P_{t a l k}$ and $\rho$ influence on probability of error

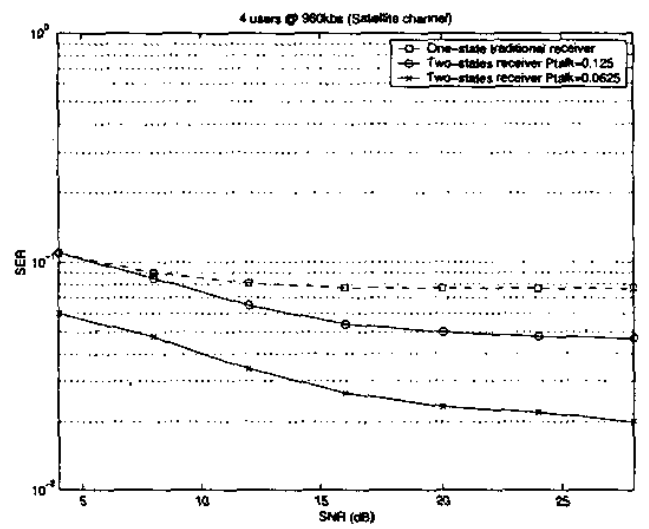

Fig. 4. Symbol Error Rate (two-state) and Bit Error Rate (conventional) and for 4 users at $960 \mathrm{kbps}\left(100 \%\right.$ of nominal system load), $P_{t a l k}$ of 0.125 and 0.0625

proposed receiver concerning the status of the transmitter. This additional information in a conventional receiver requires a signaling which has an impact on the overall performance. In this sense the results shown below are not completely fair to the proposed receiver as concerns the offered service.

In fig. 3 is reported a theoretical evaluation of the probabilities of generalized symbol error for the conventional two-states CDMA receiver, compared to the probability of error of the single-state receiver. The curves are reported for different values of the normalized cross-correlation index $(\rho)$ and different values of $P($ talk $)$ (for the two-states receiver only). As shown, the low activity region $\left(P_{\text {talk }}<0.5\right)$ is characterized by a substantial improvement of the proposed transmission scheme over the traditional "always-on" transmission.

The comparison between the proposed two-states RAKE receiver and the conventional one-state receiver has been carried out in the following summarized cases:

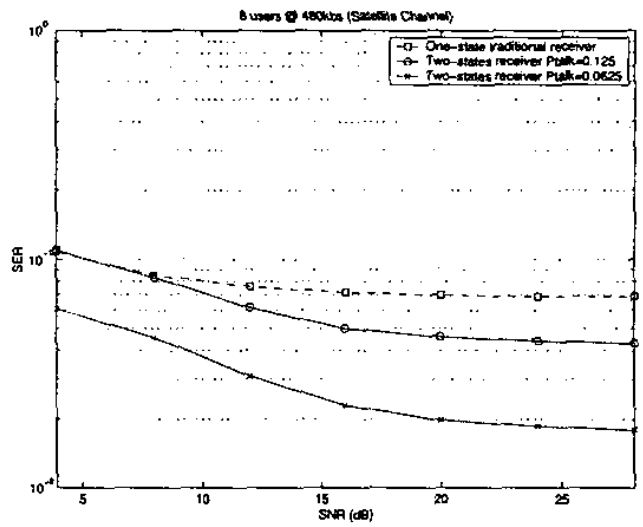

Fig. 5. Symbol Error Rate (two-state) and Bit Error Rate (conventional) and for 8 users at $480 \mathrm{kbps}\left(100 \%\right.$ of nominal system load), $P_{\text {talk }}$ of 0.125 and 0.0625

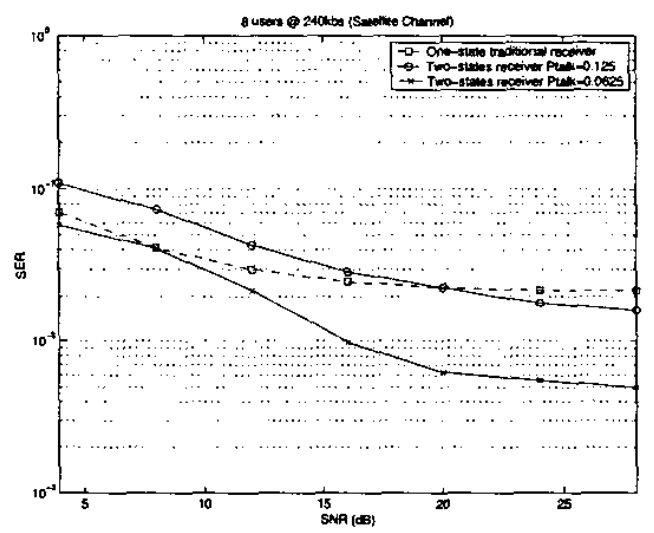

Fig. 6. Symbol Error Rate (two-state) and Bit Error Rate (conventional) and for 8 users at $240 \mathrm{kbps}$ ( $50 \%$ of nominal system load), $P_{\text {talk }}$ of 0.125 and 0.0625

1. 4 users @ $960 \mathrm{kbs}$ ( $100 \%$ of the system load) with a $P($ talk $)$ ranging from 0.125 to 0.0625 ;

2. 8 users @ $240 \mathrm{kbs}(50 \%$ of the system load) with a $P(t a l k)$ ranging from 0.125 to 0.0625 ;

3. 8 users @ $480 \mathrm{kbs}$ ( $100 \%$ of the system load) with a $P($ talk $)$ ranging from 0.125 to 0.0625 ;

In Fig. 4, 6 and $S$ are shown the ternary symbol error rate of the two-states CDMA receiver and the bit error rate for the single-state receiver. The curves are reported for different values of the SNR and different values of $P(t a l k)$ (for the two-states receiver only). All simulations assumed the same throughput for the two compared communication systems. As the probability of a talk symbol decreases, the two-states transmission method performs significantly better the standard one, since the frequent but short silences reduce the average MAI interference without any signaling overhead. 
All these results coupled with the lower power consurnption, lead us to conclude that the proposed CDMA communication scheme is able to get practical advantages over the standard proposed S-UMTS communication systems.

\section{CONCLUSIONS}

In this paper is presented a new CDMA transmission scheme based on a variable energy symbols constellation called "twostates" transmission. A theoretic analysis shows the convenient use of the proposed signaling method in CDMA systems where MAI and complexity power are the dominant limiting factors. A detailed theoretical study has been conducted to express the exact probability of error for the conventional detector. Numerical performance evaluation and comparison based on the probability of errors of the conventional one-state and two-states receivers have been reported in a real S-UMTS environment. The use of the silence symbol reduces MAI on the overall access scheme, thus allowing more users on the satellite link. Hence, the proposed communication scheme is able to get practical advantages over the traditional single-state communication scheme, especially for "burst" data transfer.

\section{REFERENCES}

[1] ETSI, "Digital cellular telecommunications systern (phase 2+); radio subsystem link control (gsm 05.08 v. 8.5.0 release 1999)," Docurrent ETSI TS 100911 v. $8.5 .0(2000-10)$.

[2] ETSI, "Digital cellular telecommunications system (phase 2+); func-tions related to mobile station $(\mathrm{ms})$ in idle mode and group receive mode (gsm 03.22 v. 8.3.0 release 1999)," Document ETSI TS 100930 v. 8.i.0 (2000$01)$.

[3] ETSI, "Gsrn phase 2+ specifications," TS-101-855.

[4] Brady P. T., "A statistical analysis of On-Off patterns in 16 conversations," Bell Syst. Tech. J., vol. 47, pp. 73-91, 1968.

[5] L. Mucchi, L. S. Ronga, and E. Del Re, "Asymptotic multiuser efficiency for a two-states cdma communication systenn" GLOBECOM'CI Conference Proceedings, San Antonio, TX, USA, 25-29 Nov. 2001.

[6] L. Mucchi, Advanced CDMA detection techniques for multipath fading wireless channels under non-ideal conditions, Ph.D. thesis, University of Florence, Florence, Italy, December 2001.

[7] C. W. Helstrom, Statistical Theory of Signal Detection, Pergamon, London, 2 nd edition edition, 1968.

[8] S-UMTS Physical Layer, "Etsi ts101-851-01," http://www.etsi.org/.

[9] Ruxandra Lupas and Sergio Verdú, "Linear multiuser detectors for synchronous code division multiple access channels," IEEE Trans. on Inf. Theory, vol. 35, no. 1, pp. 123-136, January 1989. 